PAPER

\title{
Longitudinal thalamic diffusion changes after middle cerebral artery infarcts
}

\author{
D Hervé, N Molko, S Pappata, F Buffon, D LeBihan, M-G Bousser, H Chabriat
}

See Editorial Commentary, page 159

J Neurol Neurosurg Psychiatry 2005;76:200-205. doi: 10.1136/jnnp.2004.041012

\begin{abstract}
See end of article for authors' affiliations

.....................

Correspondence to: H Chabriat, Service de Neurologie, Hôpital Lariboisière, 3 rue Ambroise Paré, 75010

Paris, France;

hugues.chabriat@

Irb.ap-hop-paris.fr
\end{abstract}

Received 22 March 2004

Revised version received 8 July 2004

Accepted 7 August 2004
Background: Cerebral infarcts are responsible for functional alterations and microscopic tissue damage at distance from the ischaemic area. Such remote effects have been involved in stroke recovery. Thalamic hypometabolism is related to motor recovery in middle cerebral artery (MCA) infarcts but little is known concerning the tissue changes underlying these metabolic changes. Diffusion tensor imaging (DTI) is highly sensitive to microstructural tissue alterations and can be used to quantify in vivo the longitudinal microscopic tissue changes occurring in the thalamus after MCA infarcts in humans.

Methods: Nine patients underwent DTI after an isolated MCA infarct. Mean diffusivity (MD), fractional anisotropy (FA), and thalamic region volume were measured from the first week to the sixth month after stroke onset in these patients and in 10 age matched controls.

Results: MD significantly increased in the ipsilateral thalamus between the first and the sixth month $\left(0.766 \times 10^{-3} \mathrm{~mm}^{2} / \mathrm{s}\right.$ first month; $0.792 \times 10^{-3} \mathrm{~mm}^{2} / \mathrm{s}$ third month; $0.806 \times 10^{-3} \mathrm{~mm}^{2} / \mathrm{s}$ sixth month). No significant modification of FA was detected. In six patients, the ipsilateral/contralateral index of MD was higher than the upper limit of the $95 \% \mathrm{Cl}$ calculated in 10 age matched controls. An early decrease of MD preceded the increase of ipsilateral thalamic diffusion in one patient at the first week and in two other patients at the first month.

Conclusion: After MCA infarcts, an increase in diffusion is observed with DTI in the ipsilateral thalamus later than 1 month after the stroke onset. This is presumably because of the progressive loss of neurons and/or glial cells. In some patients, this increase is preceded by a transient decrease in diffusion possibly related to an early swelling of these cells or to microglial activation. Further studies in larger series are needed to assess the clinical correlates of these findings.
$\mathrm{F}$ ocal cerebral infarcts may be responsible for histological and functional changes remote from ischaemic lesions. ${ }^{12}$ Several authors previously suggested that diaschisis detected with functional imaging may have a critical role in stroke recovery. ${ }^{3}$ After middle cerebral artery (MCA) infarcts, both the integrity of the corticospinal tract and the delayed thalamic hypometabolism were found related to the motor recovery. ${ }^{4}$ However, little is known concerning the microscopic tissue changes underlying the functional alterations occurring in areas remote from the infarction. Postmortem, gliosis, and neuronal loss have been repeatedly reported within the ipsilateral thalamus after MCA infarcts in animals ${ }^{15}$ but only in one case in humans. ${ }^{6}$ Non-specific thalamic signal abnormalities have been also seen on T2weighted magnetic resonance imaging (MRI) in stroke patients after these ischaemic lesions. Finally, although ipsilateral thalamic atrophy on computed tomography (CT) scan or MRI has been repeatedly reported several weeks after MCA infarcts in humans, ${ }^{67}$ both the degree and natural history of the underlying thalamic microstructural tissue changes have as yet not been described.

Diffusion tensor imaging (DTI) is a MRI technique based on the measurement of the random displacements of water molecules. Diffusion, which is a three dimensional process, is much more anisotropic in the white matter than in grey matter because of the parallel orientation of axonal membranes and myelin, which are responsible for the restriction of water movements. The DTI technique allows investigation of tissue architecture using various quantitative parameters calculated at the voxel level. ${ }^{8}$ The mean diffusivity (MD) of the tensor reflects the magnitude of water movements independent of their direction whereas the fractional anisotropy index is a measure of directional bias in the diffusion profile. DTI is sensitive enough to detect microstructural changes well beyond signal abnormalities as seen on conventional MRI. Thus, a reduction of anisotropy without significant modifications of the mean diffusivity of water molecules was detected inside the corticospinal tract, distal to MCA infarction. This may be related to demyelination or cellular infiltration observed during Wallerian degeneration. ${ }^{9}$

In this study, we aim to quantify and follow the microstructural tissue alterations within the thalamus using DTI in patients with a recent MCA infarct.

\section{MATERIAL AND METHODS \\ Subjects}

We studied nine patients with cortical brain infarction $<7$ days within the territory of the right or left MCA and without any other signal abnormalities on $\mathrm{Tl}$ and $\mathrm{T} 2$ weighted images (WI). All patients were investigated with DTI at 1 week (D7), 1 month (M1), 3 months (M3), and 6 months (M6) following a predefined protocol. Diffusion data obtained at the onset of the ischaemic stroke were not used in the present study because they were obtained in different centres with different imaging parameters and did not always include Apparent Diffusion Coefficient (ADC) maps.

\footnotetext{
Abbreviations: ADC, Apparent Diffusion Coefficient; CADASIL, cerebral autosomal dominant arteriopathy with subcortical infarcts and leucoencephalopathy; CT, computed tomography; DTI, diffusion tensor imaging; FA, fractional anisotropy; FLAIR, fluid attenuated inversion recovery; $M C A$, middle cerebral artery; $M D$, mean diffusivity; $M M S E$, Mini-Mental State Examination; MRI, magnetic resonance imaging; PET, positron emission tomography; $\mathrm{ROI}$, region of interest; $\mathrm{TE}$, echo time; TR, repetition time; Wl, weighted image
} 
Information on demographical characteristics and vascular risk factors were collected in each patient. All subjects had a detailed neurological examination 2 hours before the MRI examination, including an evaluation of cognitive deficit with the Mini-Mental State Examination (MMSE), neurological deficit with the NIH Stroke Scale, and degree of handicap with the Rankin Scale.

Ten healthy volunteers were investigated once using the same MRI protocol during the study period.

An independent institutional ethics committee approved the present study (CCPRB Bicêtre 9724) and informed consent was obtained for each participant.

\section{Magnetic resonance imaging}

Tl-WI, fluid attenuated inversion recovery (FLAIR) imaging, and diffusion WI were acquired with the use of a 1.5 Tesla MRI system (Signa General Electric Medical Systems, USA) equipped with gradient hardware allowing up to $22 \mathrm{mT} / \mathrm{m}$.

Tl-WI (inversion recovery) were acquired first in the axial plane with a spoiled gradient echo sequence (124 slices, $1.4 \mathrm{~mm}$ thick, repetition time $=10.4 \mathrm{~ms}$, echo time $=$ $2.2 \mathrm{~ms}$, inversion time $=600 \mathrm{~ms}$ ) and $24 \times 18 \mathrm{~cm}$ field of view (resolution of $0.9375 \times 0.9375 \times 1.4 \mathrm{~mm}$ ).

In each patient, FLAIR images were obtained using the following parameters: $2.8 \mathrm{~mm}$ thick, field of view $24 \times 24 \mathrm{~cm}$, resolution of $0.9375 \times 0.9375 \mathrm{~mm}$, repetition time $(\mathrm{TR})=$ $10002 \mathrm{~ms}$, echo time $(\mathrm{TE})=148 \mathrm{~ms}$.

Diffusion WI (2.8 mm thick) were obtained with an echo planar imaging sequence (single shot) sensitised to diffusion by application of gradient pulses on either side of the refocusing radio frequency pulse, in the axial plane, at 48 slice locations. For each slice location, T2-WI with no diffusion sensitisation $\left(\mathrm{B}_{0}\right)$, followed by 5 b values (incremented linearly to a maximum value of $\left.1000 \mathrm{~s} / \mathrm{mm}^{2}\right)$, were obtained in six non-collinear directions ( $x, y, z, x-y, x-z, y-z)$ using one repetition. The field of view was $24 \times 24 \mathrm{~cm}$ (resolution of $1.875 \times 1.875 \mathrm{~mm}$ ), echo time $=81.4 \mathrm{~ms}$, and repetition time $=2500 \mathrm{~ms}$.

During the period of data collection, the stability of diffusion measurements was verified on serial phantom studies.

\section{Image post-processing}

Before the tensor estimation was performed, an unwrapping algorithm was applied to the diffusion weighted data set to correct for distortions related to eddy currents induced by the large diffusion sensitising gradients. ${ }^{10}$ After this correction, the diffusion tensor parameters were calculated on a pixel-by-pixel basis. Both the mean diffusivity derived from the Trace of the diffusion tensor $(\mathrm{MD}=$ Trace $[\mathrm{D}] / 3)$ and the fractional anisotropy index (FA) were used for data analysis in this study. ${ }^{11}$

For each exam, a single examiner $(\mathrm{DH})$ delineated a region of interest (ROI) covering the total thalamus on each side. The anatomical boundaries of the thalamus were delineated on each slice. They were first verified simultaneously on coregistered TlWI. Thereafter, the medial boundaries of the nucleus were checked on each slice using the limits of cerebrospinal fluid of ventricles. The lateral limits were also verified using the FA maps, which are the best showing the medial limits of the internal capsules (fig 1).

In addition, the volume of the thalamus ipsilateral and contralateral to the ischaemic lesion was calculated in all patients for each exam. The final volume of the ischaemic lesion was also obtained on FLAIR images at M3 using the segmentation tool detailed above.

\section{Statistical analysis}

In controls, in the absence of any significant difference between the right and left thalamus for MD values (mean values:

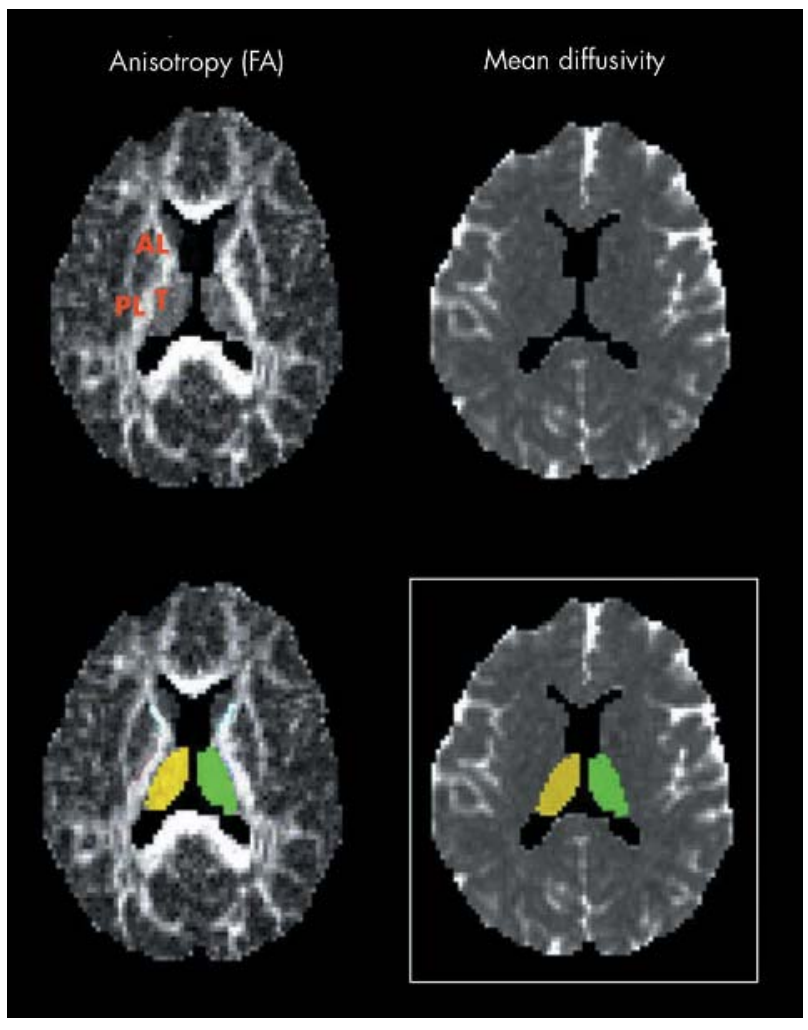

Figure 1 Fractional anisotropy (FA) and mean diffusivity (MD) maps after extraction of cerebrospinal fluid in a control subject. The lateral limits of the thalamus were verified using the fractional anisotropy maps, which are the best showing the medial limits of the internal capsules. The medial boundaries were also checked on each slice using the limits of cerebrospinal fluid of ventricles. Final result in a white rectangle. PL, posterior limb of internal capsule; AL, anterior limb of internal capsule; T, thalamus.

$0.743 \times 10^{-3} \mathrm{~mm}^{2} / \mathrm{s}$ and $0.744 \times 10^{-3} \mathrm{~mm}^{2} / \mathrm{s}$, respectively), we chose to consider only the data obtained in the right thalamus to compare absolute values in patients and controls.

To increase the regional contrasts and reduce the inter-exam variability, asymmetry indices (ipsilateral/contralateral to the ischaemic lesion) of MD values obtained in patients were compared with the right/left indices calculated in controls.

Diffusion parameters obtained at D7 in patients were first compared with those of controls with a two-tailed Student's $t$ test. In the group of patients, absolute values and asymmetry indices of MD obtained during the follow up were analysed using analysis of variance with repeated measures. Post hoc analysis was performed between different time points (D7 $v$ M1, M1 v M3, M3 v M6, and D7 v M6) based on least squares means with Tukey-Kramer correction for multiple testing.

Furthermore, for each parameter, individual data in patients were considered abnormal when they were outside of the $95 \%$ confidence interval limits of the mean value obtained in controls.

Finally, association between MD values and clinical scores were assessed using the non-parametric Spearman correlation coefficient.

Values of $\mathrm{p}<0.05$ were considered statistically significant. The statistical analysis was performed with the use of Statview software (Abacus concepts Inc., USA).

\section{RESULTS}

Among the patients, one had both hypertension and hypercholesterolaemia before admission, three had hypertension only, two were current smokers, and one had an isolated 


\begin{tabular}{|c|c|c|c|c|}
\hline & Age & Sex & Vascular risk factors & Stroke mechanism \\
\hline 1 & 69 & M & Hypertension & Cardioembolic \\
\hline 2 & 62 & M & Tóbacco use & Cardioembolic \\
\hline 3 & 60 & $\mathrm{~F}$ & $\begin{array}{l}\text { Hypertension, } \\
\text { hypercholesterolaemia }\end{array}$ & Unknown \\
\hline 4 & 49 & M & - & Arterial dissection \\
\hline 5 & 57 & $\mathrm{~F}$ & Hypercholesterolaemia & Unknown \\
\hline 6 & 78 & M & Hypertension & Atherothrombotic \\
\hline 7 & 66 & M & Hypertension & Unknown/cardioembolic \\
\hline 8 & 23 & $\mathrm{~F}$ & - & Arterial dissection \\
\hline 9 & 32 & M & Tobacco use & Atherothrombotic \\
\hline
\end{tabular}

Age did not differ between patients and controls: $55.1 \pm 15.7$ years (range: $23-78$ ) v $51.7 \pm 3.7$ years (range: $29-66$ ), respectively. F, female; $M$, male.

hypercholesterolaemia (Table 1). All patients and controls were right handed. The mean NIHSS score in patient was 5.5 at D7 (range: 2-9). At M6, the averaged Rankin score was 1.62 (range: 1-2) and MMSE score was 26.6 (range: 22-30).

\section{Location and volume of MCA infarcts}

The volume of ischaemic lesions as measured on FLAIR images at M3 ranged from 5463-102414 $\mathrm{mm}^{3}$. The cortical territory of the middle cerebral artery was involved in all subjects. The lesion also affected the deep territory of the MCA in four patients, including the lentiform nucleus $(\mathrm{n}=4)$, caudate nucleus $(\mathrm{n}=1)$, and internal capsule $(\mathrm{n}=3)$. Other details are presented in Table 2.

\section{DTI data}

Forty five examinations were obtained: 35 in patients and 10 in controls. Only one patient declined to undergo the fourth examination (M6).

At D7, no signal abnormality was detected on $\mathrm{T} 1, \mathrm{~T} 2$ weighted, and FLAIR images outside the territory of the MCA. The absolute mean value of MD calculated in the ipsilateral and contralateral thalamus in patients was significantly higher than MD measured in the thalamus in controls: ipsilateral $0.780 \times 10^{-3} \mathrm{~mm}^{2} / \mathrm{s}$ and contralateral $0.786 \times 10^{-3} \mathrm{~mm}^{2} / \mathrm{s} \quad v \quad 0.753 \times 10^{-3} \mathrm{~mm}^{2} / \mathrm{s}$ in controls $(\mathrm{p}=0.02$ and $\mathrm{p}=0.001$, respectively). In contrast, $\mathrm{MD}$ values in the whole contralateral hemisphere (after anatomic segmentation of the total hemisphere, including white and grey matter) did not differ between the two groups (fig 2).

Analysis of variance showed a significant time effect on MD measured in the ipsilateral $(p=0.005)$ but not in the contralateral thalamus. A significant increase of MD in the ipsilateral thalamus was detected between MI and M3 and

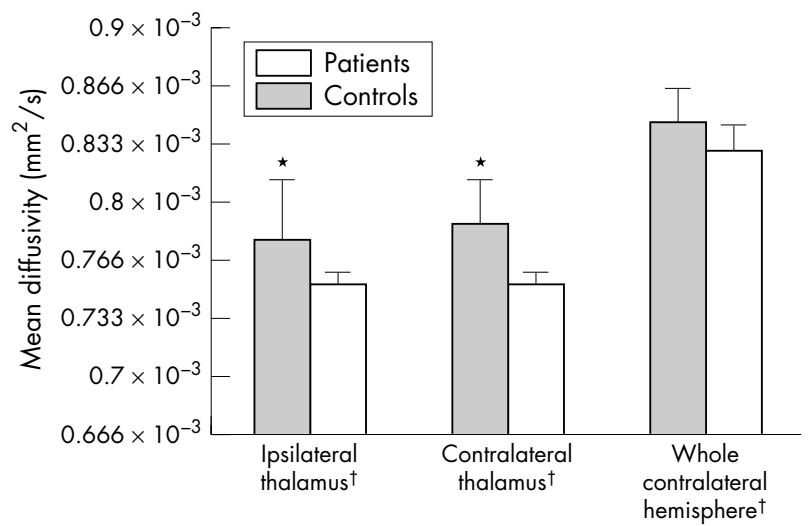

Figure 2 Comparison of mean diffusivity (MD) values between patients at D7 and controls in the ipsilateral and contralateral thalamus (right thalamus in controls) and in the whole contralateral hemisphere (whole right hemisphere in controls). ${ }^{*} \mathrm{p}<0.05$; tright side in controls.

between M3 and M6 in patients $\left(0.766 \times 10^{-3} \mathrm{~mm}^{2} / \mathrm{s}\right.$ at $\mathrm{Ml}$; $0.792 \times 10^{-3} \mathrm{~mm}^{2} / \mathrm{s}$ at $\mathrm{M} 3 ; 0.806 \times 10^{-3} \mathrm{~mm}^{2} / \mathrm{s}$ at $\left.\mathrm{M} 6\right)$. On this side, a trend toward a decreased MD was also observed between D7 and Ml that did not reach statistical significance. In contrast, no change of absolute MD values was observed in the thalamus contralateral to the lesion in patients (fig 3). Analysis of variance did not show any significant modification of FA in the ipsilateral as well as in the contralateral thalamus during the follow up (Table 3).

The analysis of thalamic asymmetry indices (I/C) during the follow up provides results identical to those obtained with absolute values. At the individual level, asymmetry indices were largely lower than the $95 \%$ confidence interval limits in three patients at D7 $(n=1)$ or Ml $(n=2)$ (figs 4 and 5). All of these patients had an ischaemic lesion involving the ipsilateral internal capsule (Table 2). Two patients presented with a small increase of the thalamic asymmetry index for MD at D7 or Ml. In contrast, asymmetry indices were significantly increased in six of eight patients at M6. There was a large decrease of the asymmetry indices in patients 2, 4, and 6 at $\mathrm{Ml}$ preceding its important increase at M3 and M6.

The asymmetry index of the thalamic volume did not differ between patients and controls at D7 (1.026 $\pm 0.66 v$ $0.963 \pm 0.072)$. No significant time effect was detected on the whole data for the thalamic volume asymmetry index. However, a trend towards a reduction in the thalamic volume was detected between D7 and M6 $(0.969 \pm 0.074 \quad v$ $0.887 \pm 0.197, \mathrm{p}=0.05)$. The asymmetry index decrease of thalamic volume between D7 and M6 was not correlated to

Table 2 Location and volume of middle cerebral artery (MCA) infarcts

\begin{tabular}{|c|c|c|c|c|c|c|c|c|}
\hline \multirow[b]{3}{*}{ Patients } & \multirow[b]{3}{*}{$\begin{array}{l}\text { Ischaemic lesion } \\
\text { volume }\left(\mathrm{mm}^{3}\right)\end{array}$} & \multicolumn{7}{|c|}{ Infarct location on FLAIR images } \\
\hline & & \multirow[b]{2}{*}{ Side } & \multicolumn{3}{|c|}{ Cortical regions } & \multicolumn{3}{|c|}{ Deep regions } \\
\hline & & & $\begin{array}{l}\text { Frontal } \\
\text { lobe }\end{array}$ & $\begin{array}{l}\text { Parietal } \\
\text { lobe }\end{array}$ & $\begin{array}{l}\text { Temporal } \\
\text { lobe }\end{array}$ & $\begin{array}{l}\text { Internal } \\
\text { capsule }\end{array}$ & $\begin{array}{l}\text { Lentiform } \\
\text { nucleus }\end{array}$ & $\begin{array}{l}\text { Caudate } \\
\text { nucleus }\end{array}$ \\
\hline 1 & 40231 & $\mathrm{R}$ & + & + & & & & \\
\hline 2 & 43992 & $\mathrm{R}$ & + & + & + & $+^{*}$ & + & + \\
\hline 3 & 102414 & $\mathrm{R}$ & + & + & & & + & \\
\hline 4 & 32898 & $\mathrm{~L}$ & + & + & & $+†$ & + & \\
\hline 5 & 31313 & $\mathrm{R}$ & & + & & & & \\
\hline 6 & 40773 & L & + & + & & & & \\
\hline 7 & 68562 & L & & + & + & & & \\
\hline 8 & 22700 & $\mathrm{~L}$ & + & + & & $+\dagger$ & + & \\
\hline 9 & 5463 & L & + & + & & & & \\
\hline
\end{tabular}




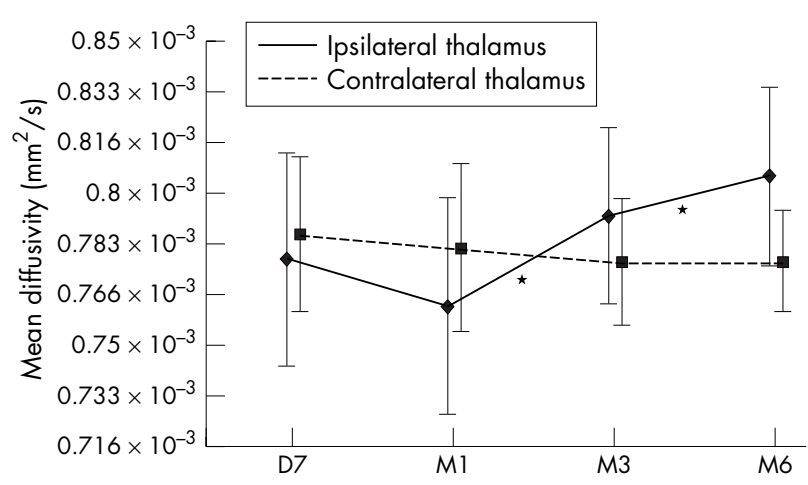

Figure 3 Longitudinal profile of mean diffusivity $(M D)$ in the ipsilateral and contralateral thalamus from D7 to M6 showing the significant modifications detected between the different sessions. *significant result of paired $t$ tests (Tukey-Kramer correction for multiple testing).

the corresponding increase of asymmetry index for $\mathrm{MD}$ during the same period.

The asymmetry index of MD calculated at M6 was not correlated to the NIHSS score obtained at the same time. There was no significant correlation between the increase of Trace (D) asymmetry indices and the variation of the NIHSS score between D7 and M6.

\section{DISCUSSION}

This is the first prospective study using DTI to monitor in vivo' the microstructural changes in the thalamus, remote from a single MCA infarct.

We observed a progressive increase in water diffusion both between M1 and M3 and between M3 and M6 in the thalamus ipsilateral to the ischaemic lesion. The mean increase between $\mathrm{Ml}$ and M6 was about 6\% in the whole group of patients. This is much higher than the $1.2 \%$ variation of MD observed in healthy subjects over the whole brain using the same technique but after a larger delay. ${ }^{12}$ At the individual level, the increase of water diffusivity was variable from 0 to $+14 \%$. These results reflect that the movements of water molecules are less restricted after 3 months in the thalamus. A cumulative loss of cell membranes, the most important barriers for the movement of water, caused by a delayed damage of glial cells or axons, is likely responsible for this finding. Increased diffusion has been already related to the loss of axons and glial cells within the cerebral white matter in leucoaraiosis or in conditions such as cerebral autosomal dominant arteriopathy with subcortical infarcts and leukoencephalopathy (CADASIL). ${ }^{13}$ Within the thalamus, a loss of neuron cells has been also reported postmortem by Ogawa et al in one patient 4 months after an acute MCA infarction. ${ }^{6}$ In animals, similar data were described by different authors. ${ }^{15}$ In the present study, the increase in diffusion was also associated with a trend toward a reduction of the global volume of the ispilateral thalamus, which is suggestive of an important loss of thalamic structural components.

Table 3 Mean values of fractional anisotropy measured in the ipsilateral and contralateral thalamus in patients with MCA infarcts

\begin{tabular}{lll}
\hline Time & $\begin{array}{l}\text { Ipsilateral thalamus } \\
\text { mean value } \pm \text { SD }\end{array}$ & $\begin{array}{l}\text { Contralateral thalamus } \\
\text { mean value } \pm \text { SD }\end{array}$ \\
\hline D7 & $0.354 \pm 0.027$ & $0.360 \pm 0.023$ \\
M1 & $0.355 \pm 0.030$ & $0.359 \pm 0.027$ \\
M3 & $0.366 \pm 0.027$ & $0.360 \pm 0.028$ \\
M6 & $0.366 \pm 0.027$ & $0.350 \pm 0.025$ \\
\hline
\end{tabular}

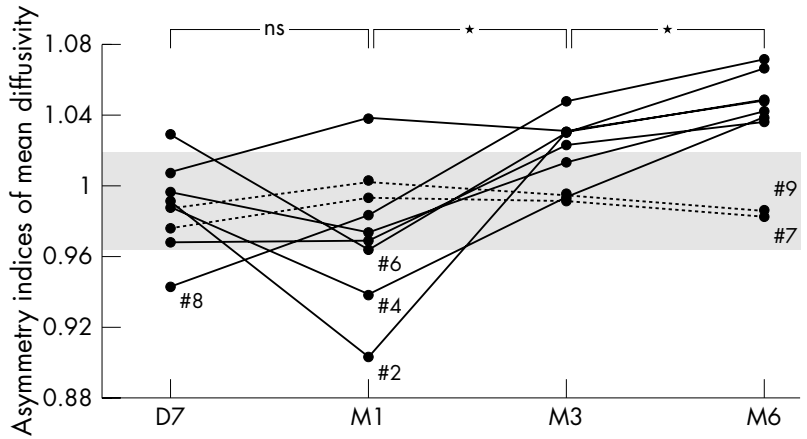

Figure 4 Individual longitudinal profiles of thalamic ipsilateral/ contralateral indices of mean diffusivity (MD) from D7 to M6.

*Significant result of paired $t$ tests (Tukey-Kramer correction for multiple testing). The $95 \%$ confidence interval of the mean value of the ipsilateral/ contralateral indices calculated in controls is presented in grey. ns, nonsignificant variation; \#, individual patient number.

This progressive atrophy of the ipsilateral thalamus has been repeatedly reported after MCA occlusion using CT scan or MRI. ${ }^{6}$

Wallerian degeneration of cortico-thalamic or thalamocortical pathways is probably responsible for the increase in diffusion and atrophy that we observed in the ipsilateral thalamus. The lack of anisotropy changes may be related to the absence of isolated bundles of parallel fibres within the thalamus. ${ }^{14}$ In CADASIL patients, Molko et al recently observed that the increase in diffusion measured in the thalamus, presumably related to a similar process, was strongly related to the degree of ischaemic lesions in the white matter. ${ }^{15}$ In the present study, we failed to find any significant correlation between the size of the final infarct and thalamic diffusion changes. Our patients presented with MCA infarcts not only of different size but also of variable topography with more or less thalamo-cortical fibres. Interestingly, two patients had no thalamic diffusion changes during the follow up study. One of them had the smallest MCA infarct of this series, the other one had a larger lesion but involving mainly the temporal cortex where thalamo-cortical

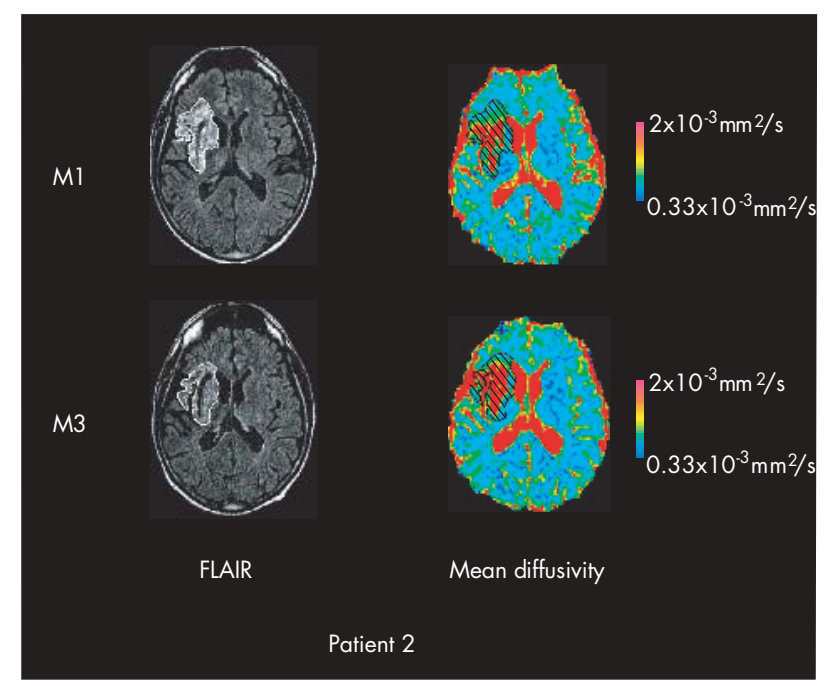

Figure 5 FLAIR and mean diffusivity (MD) images obtained in patient 2 who presented the most marked decrease of diffusion in the thalamus at M1. The ischaemic lesion is delineated with white line on FLAIR images and black lines on MD map. Note the asymmetry of diffusion with the transient decrease of $M D$ at $M 1$ in the thalamus ipsilateral to the infarct, which disappeared at M3. 
fibres are relatively few. ${ }^{16}$ In contrast, all three patients with an ischaemic lesion extending into the internal capsule had a very large increase of MD in the ipsilateral thalamus at M6. In animal models of MCA occlusion, neuronal degeneration was restricted to ventroposteromedial thalamus when the ischaemic lesion was limited to the lateral parietal cortex. ${ }^{5}$ Degeneration also involved the thalamic reticular nucleus when the infarcted area included the basal ganglia and/or the internal capsule. ${ }^{17}$ Therefore, it is possible that only the most widespread diffusion changes were detected in the thalamus by our method. Other diffusion imaging techniques, including automatic diffusion segmentation of thalamic nuclei based on the tensor data, appear promising to investigate the regional variations of diffusion within the thalamus. ${ }^{18}$

Another important result of the present study is the detection of a decrease in diffusion in the ipsilateral thalamus in one patient at D7 and in two patients at Ml. In all these patients who had a large infarct involving the cortex and the internal capsule, this decrease was transient and preceded a large increase in diffusion at M6. These data cannot be attributed to ischaemic cytotoxic oedema for several reasons: 1) the location of the ischaemic lesions as detected on late FLAIR and T2-WI only concerned the MCA territory and did not involve the thalamus; 2) the delay for the decrease in diffusion observed in these patients was not that observed in cytotoxic oedema. Such a decrease in diffusion has been already reported by Virley et al after MCA occlusion in rats in the ipsilateral thalamus at D7. ${ }^{19}$ In a similar animal model, a transient decrease preceding a large increase in diffusion has also been reported within the ipsilateral substantia nigra remote from the ischaemic area. ${ }^{20}$ This diffusion decrease was temporally related to the swelling of perivascular feet of astrocytes and neurons in this area and preceded the loss of neurons. Astrocytic and microglial activation and infiltration has already been reported during axonal degeneration in animals ${ }^{21}$ and may account for the decreased mobility of water molecules in the ipsilateral thalamus. Positron emission tomography (PET) studies using the [11C]PK11195, a marker of microglial activation, have previously demonstrated an increased fixation of this ligand within the ipsilateral thalamus. ${ }^{22}$ Whether the decrease in thalamic diffusion is caused either by the swelling of astrocytes and neurons or by microglial activation increasing the local cellularity, or by both of these phenomena, warrant further investigations. In this series, the largest diffusion decrease was detected in the patients who had their infarct spreading over the internal capsule where thalamic radiations are concentrated. ${ }^{16}$ This suggests that the number of damaged fibres leaving the ipsilateral thalamus is probably crucial in determining the microstructural modifications underlying the diffusion changes.

The thalamic metabolic activity has been already correlated to the degree of motor recovery in a series of 23 patients investigated with PET and fluoro-deoxy-glucose. ${ }^{4}$ This metabolic depression reflecting a depression of synaptic activity was thought to result from structural damage of the thalamic connections with the cerebral cortex. In the present study, we found that microstructural damage was present within the ipsilateral thalamus but did not find any significant correlation between the clinical scores and the increase in diffusion measured at the end of our follow up period. The lack of clinical correlation with diffusion changes between D7 and M6 may be related both to the low variance of the clinical scores obtained at this time and to the small size of our sample. In this study, we did neither use extensive neuropsychological batteries to evaluate the cognitive performances nor any specific assessment of motor recovery. Therefore, whether secondary microstructural tissue alterations underlying the thalamic diffusion changes are involved in some cognitive alterations or in the recovery of subtle motor performances will need further studies.

At D7, water diffusion in both thalami was found higher in patients than in controls, but later the kinetics of diffusion changes in the contralateral thalamus differs from that in the ipsilateral thalamus. In the contralateral thalamus, the high MD values were stable between D7, M1, M3, and M6. The lack of increased diffusion in the whole contralateral hemisphere at D7 suggests that the increased water mobility was mainly restricted to the thalamus on this side. Aging, which has previously been reported to increase diffusion in the normal brain, cannot account for this finding because of the very small and non-significant age difference between patients and controls. In the absence of DTI data at the onset of cerebral ischaemia, early effects of the ischaemic insult cannot be ruled out. However, they also appear very unlikely because of the complete stability of diffusion in the contralateral thalamus during the follow up. It was noteworthy that seven out of nine patients in this series had one or several vascular risk factors, including hypertension in four of them. In a model of hypertensive rats, increased diffusion has been already detected in the deep grey matter. ${ }^{23}$ Whether these vascular risk factors are responsible for a permanent increase in diffusion caused by microstructural tissue alterations in the thalamus contralateral to the infarcts is therefore an alternative hypothesis.

In conclusion, our study showed that microstructural tissue loss occurred progressively after 1 month in regions remote from cerebral infarcts. This is visible in vivo with DTI not only in the white matter ${ }^{9}$ but also in the grey matter remote from the primary insult.

\section{Authors' affiliations}

D Hervé, M-G Bousser, H Chabriat, The Department of Neurology, CHU Lariboisière, Paris, France

N Molko, S Pappata, INSERM U562, SHFJ-CEA, Orsay, France F Buffon, D LeBihan, H Chabriat, UNAF-IFR 49, SHFJ-CEA, Orsay, France

Competing interests: none declared

\section{REFERENCES}

1 Kataoka K, Hayakawa T, Yamada K, et al. Neuronal network disturbance after focal ischemia in rats. Stroke 1989;20:1226-35.

2 De Reuck J, Decoo D, Lemahieu I, et al. Ipsilateral thalamic diaschisis after middle cerebral artery infarction. J Neurol Sci 1995; 134:130-5.

3 Seitz RJ, Azari NP, Knorr U, et al. The role of diaschisis in stroke recovery. Stroke 1999;30:1844-50.

4 Binkofski F, Seitz RJ, Arnold S, et al. Thalamic metabolism and corticospinal tract integrity determine motor recovery in stroke. Ann Neurol 1996:39:460-70.

5 Lizuka H, Sakatani K, Young W. Neural damage in the rat thalamus after cortical infarcts. Stroke 1990;21:790-4.

6 Ogawa T, Yoshida Y, Okudera T, et al. Secondary thalamic degeneration after cerebral infarction in the middle cerebral artery distribution: Evaluation with MR imaging. Radiology 1997;204:255-62.

7 Tamura A, Tahira Y, Nagashima H, et al. Thalamic atrophy following cerebral infarction in the territory of the middle cerebral artery. Stroke $1991 ; 22: 615-18$.

8 Le Bihan D, Mangin JF, Poupon C, et al. Diffusion tensor imaging: concepts and applications. J Magn Reson Imaging 2001;13:534-46.

9 Werring DJ, Toosy AT, Clark CA, et al. Diffusion tensor imaging can detect and quantify corticospinal tract degeneration after stroke. I Neurol Neurosurg Psychiatry 2000;69:269-72.

10 Poupon C, Clark CA, Frouin V, et al. Regularization of diffusion-based direction maps for the tracking of brain white matter fascicles. Neuroimage 2000; 12:184-95.

11 Pierpaoli C, Jezzard P, Basser PJ, et al. Diffusion tensor MR imaging of the human brain. Radiology 1996;201:637-48.

12 Molko N, Pappata S, Mangin JF, et al. Monitoring disease progression in CADASIL with diffusion magnetic resonance imaging: a study with whole brain histogram analysis. Stroke 2002 Dec;33(12):2902-8.

13 Chabriat H, Pappata S, Poupon C, et al. Clinical severity in cadasil related to ultrastructural damage in white matter: in vivo study with diffusion tensor MRI. Stroke 1999:30:2637-43.

14 Pierpaoli C, Barnett A, Pajevic S, et al. Water diffusion changes in wallerian degeneration and their dependence on white-matter architecture. Neuro Image $2001 ; 13: 1174-85$. 
15 Molko N, Pappata S, Mangin JF, et al. Diffusion tensor imaging study of subcortical gray matter in cadasil. Stroke 2001;32:2049-54.

16 Carpenter MB. Core text of neuroanatomy. Timothy S, ed. Lippencott, Williams \& Wilkins, 1991.

17 Dihne M, Grommes C, Lutzenburg M, et al. Different mechanisms of secondary neuronal damage in thalamic nuclei after focal cerebral ischemia in rats. Stroke 2002;33:3006-11

18 Dauguet J, Cointepas D, Hervé D, et al. Eleventh meeting of the international society of magnetic resonance in medicine, 2003.

19 Virley JS, Beech SCSSCR, Williams AJ, et al. Secondary degeneration following transient mcao in the rat: Quantitative changes using MRI. The International Society for Cerebral Blood Flow and Metabolism 1999;19(Suppl 1):S493.
20 Nakane M, Tamura A, Miyasaka N, et al. Astrocytic swelling in the ipsilatera substantia nigra after occlusion of the middle cerebral artery in rats. AJNR 2001;22:660-3

21 Acarin L, Gonzalez B, Hidalgo J, et al. Primary cortical glial reaction versus secondary thalamic glial response in the excitotoxically injured young brain: astroglial response and metallothionein expression. Neuroscience 1999;92:827-39.

22 Pappata S, Levasseur M, Gunn RN, et al. Thalamic microglial activation in ischemic stroke detected in vivo by pet and [1 lc]pk1195. Neurology 2000:55:1052-4

23 Guerrini U, Sironi L, Tremoli E, et al. New insights into brain damage in stroke-prone rats: A nuclear magnetic imaging study. Stroke 2002;33:825-30

\section{HISTORICAL NOTE}

\section{Kanner's infantile autism and Asperger's syndrome}

doi: 10.1136/jnnp.2004.042820

$\mathrm{R}$ ecent much publicised attention to autism and its putative relation to the measles, mumps, and rubella (MMR) vaccination reminds us that autism affects approximately 4 in 10000 of the population. It is characterised by impairments in reciprocal social interaction and communication, restricted and stereotyped patterns of interests and activities, and the presence of developmental abnormalities by 3 years of age. Much of the psychiatric literature appears to overlook the organic basis, ${ }^{1}$ with subtle neurological signs evident in many examples: learning difficulties, a high incidence of epilepsy, viral infections, tuberous sclerosis, and fragile $\mathrm{X}$ syndrome are known associations.

Leo Kanner, a psychiatrist at Johns Hopkins, recognised children sent to his clinic with similar characteristics, which he named "early infantile autism". In his original description, Kanner ${ }^{2}$ noted that in most cases the child's behaviour was abnormal from early infancy. He therefore suggested an inborn, presumably genetic, defect. Subsequently, an increased concordance rate in monozygotic $v$ dizygotic twins and a 75 -fold increased risk to siblings of idiopathic cases were shown.

"Autistic psychopathy in childhood" by Hans Asperger ${ }^{3}$ was published in 1944 in German (translated into English in 1991). His description of autistic psychopathy in four cases is similar to Kanner's "early infantile autism" ${ }^{4}$ Symptoms described by Asperger included the DSM-IV's three diagnostic criteria for autism.

A Viennese physician, Asperger, described young boys of normal intelligence and language development, but who also exhibited autistic like behaviours and marked deficiencies in social and communication skills.

While Kanner reported that 3 of his 11 patients did not speak at all, and the remainder rarely used language, Asperger noted that his patients spoke "like little adults"; Kanner reported poor motor coordination but good fine motor skills, although Asperger observed that both were affected. He described:

"Impairment in social interaction, impairments in communication, and restricted, repetitive, and stereotyped patterns of behaviour, interests, and activities..."

Asperger's cases had:

"Special interests, abnormal fixations, stereotypic play and movements, such as rocking, and ritualized behaviours."

He described the characteristics of 11 children and he selected features crucial for diagnosis:
- a profound lack of affective (emotional) contact with other people

- intense insistence on sameness in their routines

- muteness or abnormality of speech

- fascination with manipulating objects

- high levels of visuospatial skills or rote memory but major learning difficulties in other areas

- an attractive, alert, intelligent appearance.

Most accounts include associated features, for example unusual sensory responses. Intelligence, according to Asperger's description, encompassed all levels of intelligence from "the highly original genius...to...mentally retarded individual". His publication received little attention outside Germany and was undoubtedly eclipsed by Kanner's description. $^{5}$

In England, Lorna Wing ${ }^{5}$ was the first to use the term Asperger's syndrome, in $1981 .^{6}$ Her description differed in some ways from Asperger's. She suggested that the term be used for:

"Children and adults who have autistic features, but who talk grammatically and who are not socially aloof."

It is likely that Kanner's and Asperger's syndromes are different ends of the same spectrum, with no aetiological differences. Social impairment and restricted and repetitive behaviour and interests are required DSM-IV symptoms for both autistic disorder and Asperger's disorder; but, communication impairment signifies a diagnosis of autism, not Asperger's disorder.

J M S Pearce

304 Beverley Road, Analby, Hull HU10 7BG, UK; imspearce@freenet.co.uk

\section{References}

1 Lobascher ME, Kingerlee PE, Gubbay SS. Childhood autism: an investigation of aetiological factors in twenty-five cases. $\mathrm{Br} J$ Psychiatry 1970;117:525-9

2 Kanner L. Autistic disturbances of affective contact. Nerv Child 1943;2:217-50.

3 Asperger H. Die 'Autistischen psychopathen' im kindesalter. Arch Psychiatr Nervenkr 1944;117:76-136.

4 Wing L. The relationship between Asperger's syndrome and Kanner's autism. In: Frith U, eds. Autism and Asperger syndrome. Cambridge: Cambridge University, 1991:93-121.

5 Wing L. The history of Asperger syndrome. In: Schopler E, Mesibov GB, Kunce $\sqcup$, eds. Asperger syndrome or high-functioning autism? New York: Plenum, 1998:11-28.

6 Wing L. Asperger's syndrome: a clinical account. Psychol Med $1981 ; 11: 115-29$ 